\title{
Can One Both Contribute to and Benefit from Herd Immunity?
}

\author{
LUCIE WHITE \\ Utrecht University
}

\begin{abstract}
In a recent article, "Vaccine Refusal Is Not Free Riding", Ethan Bradley and Mark Navin (2021) provide us with several reasons to doubt that vaccine refusal is a free rider problem. Here, I defend one connection between vaccine refusal and free riding and suggest that, when viewed in conjunction with their other arguments, this might constitute a reason to mandate Covid-19 vaccination.
\end{abstract}

Keywords: fairness, free riding, game theory, public good, public health, vaccination

JEL Classification: C72, I18

In their recent, illuminating contribution to the ethics and economics of Covid-19, Ethan Bradley and Mark Navin (2021) provide us with several reasons to doubt the received view that we can essentially view vaccine refusal as a free rider problem. Bradley and Navin contend that from both the subjective perspective of those who refuse vaccines, and also viewed objectively, there are several important differences between vaccine refusal and classic free riding. In making these distinctions, they draw attention to differences between the subjective views of many vaccine refusers and the views that we would expect to see among free riders, with important implications for how we should go about addressing the problem of vaccine refusal. However, their argument that vaccine refusers cannot be thought of as free riders in an objective sensebecause it is not possible to both contribute to and benefit from the

AuthoR's Note: Many thanks to Philippe van Basshuysen, Donal Khosrowi, and JanFelix Müller for their-as always-incisive and insightful feedback. My sincere thanks also to an anonymous reviewer for their very constructive comments and, particularly, for pressing me on some essential distinctions. This research was made possible by the financial support of the Volkswagen Foundation. 
public good of herd immunity ${ }^{1}$-does not go through, particularly when it comes to Covid-19. Drawing this out can help us to better understand the various goals of a vaccination programme against Covid-19. Defending this particular parallel between vaccine refusers and free riders is also important because, in combination with the other arguments provided by Bradley and Navin, it says something about how we (in both a moral and a practical sense) should go about dealing with the problem of Covid-19 vaccine refusal.

\section{The Argument Against Viewing Vaccine Refusal as Free Riding}

The existence of 'public goods' - that is, goods that are non-rivalrous and non-excludable-gives rise to the free rider problem. Because these goods are non-excludable, individuals may benefit from them whether or not they contribute to their provision. The fact that it is thus rational to benefit from a public good without contributing to it-that is, to be a 'free rider'-coupled with the fact that if enough people refuse to contribute, the conditions for the existence of these goods are undermined, is the 'free rider problem'. As Bradley and Navin $(2021,170)$ note, mass vaccination creates the public good of herd immunity. Although no country has yet passed the threshold for herd immunity, we can hope that current vaccination programmes will soon allow it to be achieved (at least in some places). Even where a fixed threshold has not been achieved, however, we might argue that vaccination still contributes to a public good, by slowing the spread (and resultant consequences of infection) to some degree-herd immunity, in other words, is not an all-ornothing proposition (see Yates 2021). ${ }^{2}$ The non-vaccinated benefit from herd immunity, because herd immunity makes outbreaks of the disease, and thus one's chances of getting infected, more unlikely, even in the absence of individual protection.

\footnotetext{
${ }^{1}$ For good reason, Bradley and Navin $(2021,168 n 1)$ prefer the term 'community protection' to 'herd immunity'. Although I agree with their reasons, here, I am using the more widely recognized term.

${ }^{2}$ In addition, even if we accept that there is no public good in existence until we reach a certain threshold for herd immunity, we might still posit that there is an obligation to bring this public good into existence. While this would not be an obligation to avoid free riding (which would seem to already require the existence of a public good that some individuals are unjustly benefitting from), some argue that a duty of fairness obligates us to contribute to the creation of the public good of herd immunity (see Navin 2013; Giubilini, Douglas, and Savulescu 2018). This would still suggest that individuals can be held accountable for unjustly refusing to contribute to the benefit of herd immunity, which should suffice to support this paper's conclusion about how we should deal with the problem of vaccine refusal.
} 
It would seem, then, to be a straightforward matter to conclude that individuals who enjoy the benefits of herd immunity without having participated in mass vaccination campaigns are free riders. Bradley and Navin, however, provide us with two strands of argument against this conclusion. First, they contend that vaccine refusers "do not have the subjective beliefs and attitudes of free riders" (2021, 171). In order to be free riders in this subjective sense, Bradley and Navin argue, vaccine refusers must acknowledge that they are indeed benefitting from the public good in question, and they must recognise that they are refusing to make some reasonable contribution towards this public good. Both of these attitudes, Bradley and Navin point out, are not characteristic of vaccine refusers. Vaccine refusers often both hold vastly overblown beliefs about the risks of vaccination (so that they do not view the costs of vaccination as a 'reasonable' contribution) and believe that there are no benefits to mass vaccination (thus denying that it produces a public good). It is important to point out the subjective differences between a 'classic' free rider and a 'classic' vaccine refuser because, as Bradley and Navin point out, it has implications for appropriate public policy responses. If - as would be the case with the classic free rider-an individual already believes that herd immunity is valuable and beneficial, and that the costs of contributing to this are not prohibitive, relatively minor changes to the individual's incentive structure (in the form of either rewards or punishments) could lead them to view contribution to the public good as indeed in their best interests. Where individuals believe that the costs are extremely high, and no good will be produced as a result of their contribution, this strategy is not likely to yield the same results (we will return to the further significance of this presently).

The second strand of argument revolves around the objective criteria for free riding. Here, Bradley and Navin focus more on the moral obligation to contribute to a public good (rather than on what might motivate people to do so effectively). Where individuals are free riders, the authors note, they are refusing to contribute to something that they are benefitting from, and thus should also be contributing to. ${ }^{3}$ But, Bradley and Navin contend, it is not possible to both contribute to and benefit from the public good of herd immunity. One can contribute to herd immunity through possessing individual immunity (which one can gain either by being vaccinated, or by contracting and recovering from a disease). But once a person has individual immunity, they need not (indeed,

\footnotetext{
${ }^{3}$ Bradley and Navin also provide another argument here-we will return to this below.
} 
cannot) rely on herd immunity for protection. Because it is not possible to both contribute to and benefit from the public good of herd immunity, we cannot accuse vaccine refusers of behaving in an unjust manner by benefitting from something that they should also be contributing to.

\section{The BENEFITS OF CONTRIBUTING TO COVID-19 HeRd IMMUNITY}

This argument, however, oversimplifies the benefits that herd immunity, particularly against Covid-19, confers on each member of the community. One of the primary challenges of Covid-19, and the chief goals of public policy, has been to prevent hospital systems from becoming overwhelmed (Giubilini, Savulescu, and Wilkinson 2021; Johnson et al. 2020). The additional strain on healthcare systems (even when they are still functioning to a degree) during the pandemic has led to severe delays and disruptions in accessing needed medical care for unrelated conditions (see, for example, The Lancet Rheumatology 2021; Riera et al. 2021). Vaccination against Covid-19 does not confer any protection against contracting an unrelated illness and finding oneself unable to access medical care. Thus, in contributing to herd immunity by being vaccinated against Covid-19, each individual contributes to something that he also benefits from-a functioning healthcare system.

One might solve this problem by making the good at stake excludable-limiting healthcare access only to those who have contributed to the maintenance of the healthcare system by being vaccinated-but there are very good moral reasons not to exclude people from access to healthcare (see Feinberg 1986). If we treat access to healthcare as a nonexcludable good, it generates a problem akin to the free rider problemeach individual benefits from its existence, and a widespread failure to contribute to its maintenance (by being vaccinated) will undermine the conditions for its existence. Although healthcare resources are, unlike public goods, rivalrous (too many individuals failing to vaccinate will lead to an overconsumption of limited healthcare resources, undermining the functioning of the system)-the essential parallel here remains. A functioning healthcare system is something that each individual can benefit from and contribute to, and there is thus plausibly an obligation to contribute to its maintenance through being vaccinated against Covid-19.

A second benefit that herd immunity confers on the community is an absence of the need for restrictions on the general population, which we have seen to varying degrees in many countries over the course of 
the pandemic. These have included restrictions on the number of people who can meet in public or private, requirements to wear masks in certain spaces, the closure of or restrictions on the operation of businesses, the closure of schools and workplaces, and restrictions on international movement (Askitas, Tatsiramos, and Verheyden 2021). Some of these restrictions could be conditioned on vaccination status (that is, they are excludable)-being admitted to a foreign country, for example, or being able to eat at a restaurant, might be made contingent on showing proof of vaccination rather than restricted for all. But many of these restrictions-for example, most of those imposed in the UK until July 2021 (BBC News 2021) - were not made contingent on vaccination status despite high vaccination rates, perhaps due to the difficulty of checking the vaccination status of every unmasked person in a crowded area, or person in a group above a certain size. Where governments deem it necessary to impose general restrictions on the population in order to stop the spread of Covid-19, being vaccinated contributes to conditions that allow for the lifting of such restrictions, and this provides benefits for every member of the population.

A third way in which one can contribute to and benefit from a mass vaccination programme stems from the fact that high levels of vaccination reduce the probability of viral variants arising. If sustained transmission of Covid-19 is not contained, the likelihood of viral mutation increases. This can lead to vaccinations becoming less effective, and could even result in the emergence of a vaccine-resistant strain of the virus (Rubin 2021). In being vaccinated, therefore, you are contributing not just to herd immunity for the virus through your individual immunity; you are also contributing to the prevention of variants that you may not have individual protection against. In this way, one can both contribute to, and benefit from, the public good of herd immunity.

\section{How SHOULD WE RESPOND TO VACCINE REFUSAL?}

Drawing out the ways in which individuals can benefit from herd immunity to Covid-19, while contributing to this public good through being vaccinated, highlights the various and vital goals of Covid-19 mass vaccination programmes. The benefits of herd immunity through mass vaccination are not limited to the protection of the population against infection and the adverse side effects of Covid-19, but include access to a functioning healthcare system, a lack of ongoing restrictions, and protection against the emergence of future variants. 
But preserving the argument that vaccine refusers are benefiting from something that they can and should also be contributing to also lends credence to the contention that vaccine refusers may be morally culpable for refusing to contribute to herd immunity through vaccination. If we think that vaccine refusers can be held responsible for their refusal to contribute despite the fact that they may hold false beliefs about vaccination (as do, for example, Brennan 2018 and Giubilini, Douglas, and Savulescu 2018), this might support incentivising or even compelling individuals to contribute to the goal of herd immunity (where the costs of doing so are reasonable-see Bradley and Navin 2021, 176). This is bolstered by another argument Bradley and Navin offer against viewing vaccination as a free rider problem in an objective sense-they claim that "free riding is individually rational, but vaccine refusal is not". That is, they contend, because serious complications from vaccines are exceedingly rare, "it is almost always in a person's interest to vaccinate, even when community protection makes their odds of infection very low" (2021, 173). In incentivizing or mandating vaccination, we would therefore not be imposing unreasonable burdens on the individual-in fact, each individual would be likely to benefit from this, beyond the benefits entailed by herd immunity.

Reintroducing Bradley and Navin's arguments against viewing vaccine refusal as free riding in a subjective sense might further steer our sense of what could constitute an appropriate and effective policy response. To recap, Bradley and Navin suggest that because vaccine refusers often do not see mass vaccination as producing any benefit, and because they believe the individual costs of vaccination are very high, our typical response to classic free rider problems-introducing incentives to contribute to the agreed-upon public good-is not likely to work effectively here. This might be thought to point us, at least prima facie, ${ }^{4}$ in the direction of mandating, rather than incentivizing, vaccination where we have problems achieving or approaching the public good of herd immunity.

Scrutiny of Bradley and Navin's arguments is thus a useful exercise in considering what constitutes effective and justifiable vaccination pol-

\footnotetext{
${ }^{4}$ This is certainly not to say that this alone is sufficient to point us in this directionseveral practical considerations may speak against such a policy. To take just one example, compulsory vaccination policies could lead vaccine refusers to avoid seeking medical care for themselves and their children (Flanigan 2014). For a comprehensive defense of vaccine compulsion, including sustained discussion of such practical considerations, see Flanigan (2014) and Giubilini (2020).
} 
icy for Covid-19. I have argued, against Bradley and Navin, that the ethical argument that Covid-19 vaccine refusers are unjustly refusing to contribute to the benefit of herd immunity retains its force once we take a broader view of the resultant benefits. Vaccine refusers might thus be morally culpable for failing to contribute to the various significant benefits that herd immunity to Covid-19 provides. Coupled with Bradley and Navin's compelling arguments that incentivizing vaccination may be of limited use, and that vaccine refusal is rarely in the best interests of the individual, this could be viewed as lending support to the case for Covid-19 vaccine mandates where the public good of herd immunity cannot be achieved through other means.

\section{REFERENCES}

Askitas, Nikolaos, Konstantinos Tatsiramos, and Bertrand Verheyden. 2021. "Estimating Worldwide Effects of Non-Pharmaceutical Interventions on COVID-19 Incidence and Population Mobility Patterns Using a Multiple-Event Study." Scientific Reports 11: 1972.

BBC News. 2021. “Covid Rules: What Has Changed?" BBC News. Accessed 27 July, 2021. https://www.bbc.com/news/explainers-52530518.

Bradley, Ethan, and Mark Navin. 2021. "Vaccine Refusal Is Not Free Riding." Erasmus Journal of Philosophy and Economics 14 (1): 167-181.

Brennan, Jason. 2018. "A Libertarian Case for Mandatory Vaccination." Journal of Medical Ethics 44 (1): 37-43.

Feinberg, Joel. 1986. The Moral Limits of the Criminal Law. Volume 3: Harm To Self. New York, NY: Oxford University Press.

Flanigan, Jessica. 2014. "A Defense of Compulsory Vaccination.” HEC Forum 26 (1): 525.

Giubilini, Alberto. 2020. "An Argument for Compulsory Vaccination: The Taxation Analogy." Journal of Applied Philosophy 37 (3): 446-466.

Giubilini, Alberto, Thomas Douglas, and Julian Savulescu. 2018. "The Moral Obligation to Be Vaccinated: Utilitarianism, Contractualism, and Collective Easy Rescue." Medicine, Health Care and Philosophy 21 (4): 547-560.

Giubilini, Alberto, Julian Savulescu, and Dominic Wilkinson. 2021. "Queue Questions: Ethics of COVID-19 Vaccine Prioritization.” Bioethics 35 (4): 348-355.

Johnson, Helen C., Céline M. Gossner, Edoardo Colzani, John Kinsman, Leonidas Alexakis, Julien Beauté, Andrea Würz, Svetla Tsolova, Nick Bundle, and Karl Ekdahl. 2020. "Potential Scenarios for the Progression of a COVID-19 Epidemic in the European Union and the European Economic Area, March 2020." Eurosurveillance 25 (9): 8-12.

Navin, Mark. 2013. "Resisting Moral Permissiveness About Vaccine Refusal." Public Affairs Quarterly 27 (1): 69-85.

Riera, Rachel, Ângela Maria Bagattini, Rafael Leite Pacheco, Daniela Vianna Pachito, Felipe Roitberg, and Andre Ilbawi. 2021. "Delays and Disruptions in Cancer Health 
Care Due to COVID-19 Pandemic: Systematic Review." JCO Global Oncology 7: 311323.

Rubin, Rita. 2021. "COVID-19 Vaccines vs Variants-Determining How Much Immunity Is Enough.” JAMA 325 (13): 1241-1243.

The Lancet Rheumatology. 2021. "Editorial-Too Long to Wait: The Impact of COVID-19 on Elective Surgery." The Lancet Rheumatology 3 (2): E83.

Yates, Kit. 2021. "(How) Can We Reach Herd Immunity." The BMJ Opinion, July 16, 2021. https://blogs.bmj.com/bmj/2021/07/16/kit-yates-how-can-we-reach-herdimmunity/.

Lucie White is an assistant professor at Utrecht University, specializing in bioethics. She is currently working on a couple of projects which focus, in various ways, on policy responses to Covid-19.

Contact e-mail: <l.a.white@uu.nl> 